\title{
DOUBLE POINTS ON HYPERBOLIC SURFACES
}

\author{
TROELS JØRGENSEN AND HANNA SANDLER
}

(Communicated by Clifford J. Earle, Jr.)

\begin{abstract}
It is shown that every proper intersection point of two closed geodesics on an orientable hyperbolic surface is either a simultaneous double point of two closed geodesics of equal length or a quadruple point, and this phenomenon persists under deformations of the surface.
\end{abstract}

\section{INTRODUCTION}

A double point on a hyperbolic surface is a point at which a closed geodesic properly self-intersects. A quadruple point is a point through which a single closed geodesic passes at least four times in different directions. Given a hyperbolic surface, we use the term "permanent" for properties which persist under arbitrary deformations of the surface. We prove:

Theorem. On any orientable hyperbolic surface, a point which is a proper intersection point of two distinct closed geodesics is either a permanent simultaneous double point of two closed geodesics of permanently equal length or a permanent quadruple point.

\section{Closed geodesics}

Throughout this paper, except in the last section, all hyperbolic surfaces are assumed to be orientable. Such a surface can be thought of as the quotient space of a hyperbolic plane by a certain group of orientation-preserving isometries of hyperbolic 3-space which leaves the plane invariant and preserves its orientation. The group acts freely and discontinuously on the invariant plane and may be identified with a discrete subgroup of $\operatorname{SL}(2, \mathbb{C})$. The closed geodesics on the hyperbolic surface correspond to (conjugacy classes of) axes of the associated covering transformations. In order to determine whether two closed geodesics are equal in length, we use the fact that the trace of $X \in \mathrm{SL}(2, \mathbb{C})$, denoted $\tau_{X}$, determines the length of the associated geodesic. Indeed, the trace is twice the hyperbolic cosine of half the length of the geodesic.

Suppose $X_{1}$ and $X_{2}$ are variable elements of $\operatorname{SL}(2, \mathbb{C})$ and $W\left(X_{1}, X_{2}\right)$ is a word in $X_{1}$ and $X_{2}$. We make use of the fact that the trace $\tau_{W\left(X_{1}, X_{2}\right)}$ is a polynomial in $\tau_{X_{1}}, \tau_{X_{2}}$, and $\tau_{X_{1} X_{2}}$ with integer coefficients (see $[1,3]$ ).

Received by the editors August 12, 1991 and, in revised form, March 19, 1992.

1991 Mathematics Subject Classification. Primary 30F99, 51M10, 53A35.

Research of the second author was supported in part by NSF grant \# DMS-9009521. 


\section{PALINDROMES}

Given two elements $X, Y \in \operatorname{SL}(2, \mathbb{C})$, having no common fixed points on the Riemann sphere, the Lie product $X Y-Y X$ determines a Möbius transformation whose axis is the common perpendicular of the axes of $X$ and $Y$. Furthermore, the axis of the Lie product is perpendicular to the axes of all nontrivial palindromes in $X$ and $Y$. (See [2].)

Let $X$ and $Y$ be elements of $\operatorname{SL}(2, \mathbb{C})$ with no common fixed points on the Riemann sphere. In the following discussion we will assume that $X$ and $Y$ generate a free group of rank two, since this is the case we will need for the proof of our theorem in the next section. The common perpendicular of the axes of any such pair $X$ and $Y$ is also the common perpendicular of the axes of two elements $A$ and $B$ of equal trace which generate a free group of rank two. For instance, we may let $A=X Y^{2} X$ and $B=Y X^{2} Y$. (The construction we give below would work for any pair of elements $A$ and $B$ of equal trace which generate a free group of rank two. For the proof that follows in the next section, we require that the axes of $A$ and $B$ have the same common perpendicular as the axes of $X$ and $Y$.) We consider the following conjugates of $A^{2} B^{2} A^{-2} B^{-2}$ :

$$
\begin{array}{ll}
S_{1}=A B^{2} A^{-2} B^{-2} A, & T_{1}=B A^{-2} B^{-2} A^{2} B, \\
S_{2}=A^{-1} B^{-2} A^{2} B^{2} A^{-1}, & T_{2}=B^{-1} A^{2} B^{2} A^{-2} B^{-1} .
\end{array}
$$

If we define

$$
W(U, V)=U V^{2} U^{-2} V^{-2} U^{2} V^{-2} U^{-2} V^{2} U,
$$

then we have

$$
\begin{array}{ll}
S_{1} S_{2}^{-1}=W(A, B), & S_{2}^{-1} S_{1}=W\left(A, B^{-1}\right), \\
T_{1} T_{2}^{-1}=W\left(B, A^{-1}\right), & T_{2}^{-1} T_{1}=W(B, A),
\end{array}
$$

and all of these words are palindromes in $A$ and $B$. Therefore, the axes of these elements are all perpendicular to the axis of $A B-B A$. Furthermore, the axes of these four elements are distinct. Indeed, if this were not the case then two of the words, being of equal trace as is shown below, would have to be identical or inverses. But, by inspection, this does not occur, since the inverse of a palindrome is obtained by inverting the exponents of the letters.

The equality of the traces is seen as follows. Observe that

$$
\begin{aligned}
\tau_{S_{1} S_{2}^{-1}} & =\tau_{W(A, B)}=P\left(\tau_{A}, \tau_{B}, \tau_{A B}\right), \\
\tau_{T_{2}^{-1} T_{1}}=\tau_{W(B, A)} & =P\left(\tau_{B}, \tau_{A}, \tau_{B A}\right),
\end{aligned}
$$

where $P$ is a polynomial with integer coefficients. Since $\tau_{A}=\tau_{B}$ and $\tau_{A B}=$ $\tau_{B A}$, and, more generally, conjugate elements have equal trace, it follows that

$$
\tau_{S_{2}^{-1} S_{1}}=\tau_{S_{1} S_{2}^{-1}}=\tau_{T_{2}^{-1} T_{1}}=\tau_{T_{1} T_{2}^{-1}} .
$$

\section{Proof of the Theorem}

Let $G$ be a surface group represented in $\operatorname{SL}(2, \mathbb{C})$, and let $S$ be the associated surface. Suppose $A$ and $B$ are elements of $G$ with intersecting axes and 
with no common fixed points on the Riemann sphere. We may as well assume (after replacing $A$ and $B$ by sufficiently high powers) that these elements generate a free group of rank two, $F_{2}$. As mentioned above, we may also assume that $A$ and $B$ have equal trace.

The group $F_{2}$, generated by $A$ and $B$, may be viewed as the covering group of an open surface of genus one which we denote by $\widetilde{S}$. Then $\widetilde{S}$ covers $S$ and, on both of these surfaces, the elements $A$ and $B$ give rise to distinct properly intersecting closed geodesics. It will be enough to prove that the intersection point of the axes of $A$ and $B$ corresponds to a permanent simultaneous double point of two distinct closed geodesics of permanently equal length on $\widetilde{S}$. Indeed, if this is the case, then the intersection point on $\widetilde{S}$ projects to a multiplicity four intersection point on $S$. We will argue below that the latter intersection point must arise from either a permanent simultaneous double point of two distinct closed geodesics of permanently equal length or from a single permanent quadruple point.

The axes of $A$ and $B$ belong to a common hyperbolic plane. Moreover, the axis of $A B-B A$ meets this plane orthogonally at the point of intersection of the axes of $A$ and $B$. Since the axes of $S_{1} S_{2}^{-1}, S_{2}^{-1} S_{1}, T_{1} T_{2}^{-1}$, and $T_{2}^{-1} T_{1}$ also belong to this plane and are perpendicular to the axis of $A B-B A$, it follows that all of these axes pass through the intersection point of the axes of $A$ and $B$. (These statements follow from [2].)

For a given surface group, two distinct axes of elements in the group will correspond to the same closed geodesic precisely when they are conjugate in the group. Therefore, the axes associated with the transformations determined by the pair $S_{1} S_{2}^{-1}$ and $S_{2}^{-1} S_{1}$ and the pair $T_{1} T_{2}^{-1}$ and $T_{2}^{-1} T_{1}$ project onto $\widetilde{S}$ as two distinct closed geodesics of equal length. This follows from the trace equivalence in equation $(*)$ and the fact that $S_{1} S_{2}^{-1}$ and $S_{2}^{-1} S_{1}$ are conjugate, as are $T_{1} T_{2}^{-1}$ and $T_{2}^{-1} T_{1}$.

Hence, on $\widetilde{S}$ the two closed geodesics, which come from the projections of the axes of $S_{1} S_{2}^{-1}, S_{2}^{-1} S_{1}, T_{1} T_{2}^{-1}$, and $T_{2}^{-1} T_{1}$, have a double point at the point of intersection of the closed geodesics determined by the axes of $A$ and $B$. As we deform the open surface of genus one and vary the representation of $F_{2}$ in $\operatorname{SL}(2, \mathbb{C})$, thereby varying $A$ and $B$, the intersection point of their axes remains a double point of the two closed geodesics of equal length associated to the elements $S_{1} S_{2}^{-1}, S_{2}^{-1} S_{1}, T_{1} T_{2}^{-1}$, and $T_{2}^{-1} T_{1}$.

We have produced a permanent simultaneous double point of two distinct closed geodesics of permanently equal length on $\widetilde{S}$. If the elements $S_{1} S_{2}^{-1}$, $S_{2}^{-1} S_{1}$, and $T_{1} T_{2}^{-1}$ become conjugate in $G$, then the simultaneous double point on $\widetilde{S}$ projects to a quadruple point on $S$; otherwise, it projects to a simultaneous double point. To deduce the desired permanence properties on $S$ from the corresponding properties on $\widetilde{S}$, we use the fact that every representation of $G$ restricts to a representation of $F_{2}$.

\section{FinAl REMARKS}

We observe that the above construction can also be carried out when the axes of $A$ and $B$ do not intersect. In this case, the common perpendicular to the axes of $A$ and $B$ lies in the same plane as these axes. The surface 
corresponding to the free group generated by $A$ and $B$ is then a three-holed sphere. Instead of obtaining two closed geodesics with a simultaneous double point on this surface, we obtain two closed geodesics of permanently equal length each of which permanently intersects the common perpendicular twice at right angles.

These results have a natural interpretation in the setting of orientable hyperbolic 3-manifolds (or orbifolds). Consider a geodesic line $l$ in a hyperbolic 3-manifold. Suppose $l$ meets arcs of closed geodesics orthogonally in two or more ways. Then we expect that our methods can be used to show that there exists a pair of closed geodesics of permanently equal length such that $l$ permanently intersects each of these geodesics orthogonally twice.

It is also worth noting that the above construction provides us with a solution to the problem of finding three elements $X_{1}, X_{2}$, and $X_{3}$ in a surface group $G$ having equal trace and satisfying $\tau_{X_{1} X_{2} X_{3}}=\tau_{X_{3} X_{2} X_{1}}$ for all representations of $G$ in $\operatorname{SL}(2, \mathbb{C})$. This equation, specialized to conjugate elements, arises when one searches for permanent triple points. (A triple point is a point through which a single closed geodesic passes at least three times in different directions.)

Typically, one expects that our construction will yield simultaneous double points, as opposed to quadruple points. However, our methods, when applied to nonorientable surfaces, can be used to produce permanent quadruple points as follows. Consider three disjoint geodesics in a hyperbolic plane. Let $\alpha, \beta$, and $\gamma$ be elliptic elements of order two corresponding to half rotations about these lines. Using the above arguments, with $A=\alpha \beta \gamma$ and $B=\beta \gamma \alpha$, we obtain four conjugate elements with distinct axes and a common point of intersection.

\section{BIBLIOGRAPHY}

1. R. Fricke and F. Klein, Vorlesungen über die Theorie der Automorphen Funktionen, Vol. 1, Teubner, Leipzig, 1897.

2. T. Jørgensen, Closed geodesics on Riemann surfaces, Proc. Amer. Math. Soc. 72 (1978), 140-142.

3. H. Vogt, Sur les $P_{n}$ varients fundamentaux des equations differentielles lineaires du second ordre, Ann. Sci. École Norm. Sup. (3) 6 3-72.

Department of Mathematics, Columbia University, New York, New York 10027

E-mail address: tj@shire.math.columbia.edu

Department of Mathematics \& Statistics, The American University, Washington, D.C. 20016

E-mail address: sandler@american.edu 